\title{
SToRytelliing to Improve Disease outcomes in Gout (STRIDE-GO) in African American veterans with gout: a trial study protocol
}

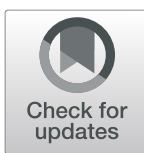

Jasvinder A. Singh ${ }^{1,2,3}$ (D

\begin{abstract}
Objective: Medication adherence in gout is suboptimal, and the lack of effective interventions to address it presents a huge challenge. Medication adherence and gout outcomes are worse in racial/ethnic minorities. The objective of this paper was to provide the details of the study protocol for randomized, controlled trial (RCT) in African Americans (AAs) with gout that will test the effectiveness of a culturally appropriate gout storytelling intervention.
\end{abstract}

Methods: The SToRytelliing to Improve Disease outcomes in Gout (STRIDE-GO) study will be a 12-month, multicenter, open-label RCT that will assess the effect of a culturally appropriate gout storytelling in at least $300 \mathrm{AA}$ veterans with gout. Participants will be randomized to gout-storytelling intervention vs. a stress reduction video in a 1:1 ratio. The primary outcome is urate-lowering therapy (ULT) adherence measured with MEMSCap ${ }^{\mathrm{TM}}$, an electronic monitoring system (efficacy, 6 months; sustenance of efficacy, 12 months). Secondary outcomes include gout flares, serum urate (SU), gout-specific health-related quality of life [HRQOL], self-reported ULT adherence, patient satisfaction with treatment, and patient understanding of the intervention. AA veterans with gout who met the 1977 Preliminary American College of Rheumatology (ACR) classification criteria for gout, currently prescribed an oral ULT medication (allopurinol or febuxostat) for at least 6 months, and not using a pillbox to redistribute their medications, will be invited to an in-person study visit. After the study coordinators obtain informed consent, and ensure that participants meet the inclusion criteria, the eligible participants will be provided with their current ULT in a MEMSCap ${ }^{\mathrm{TM}}$ bottle for the 1-month run-in period and asked to return to the clinic in 1 month. ULT adherence with MEMSCap ${ }^{\mathrm{TM}}$ will be recorded at a 1-month return visit. Interested participants will complete the baseline assessments, randomized using the computerized system to either gout-storytelling intervention or a stress reduction intervention video arm and watch the respective video in-clinic. Patients will be interviewed on the phone at 2 and 4 months regarding the viewing of the videos at home at each time. Participants will be assessed in-clinic at 3, 6, 9, and 12 months; MEMSCap ${ }^{\mathrm{TM}}$ data and patient surveys will be captured at each visit. For any missed visit, assessments will be completed on the phone and MEMSCap ${ }^{\text {TM }}$ data captured at the next in-clinic visit.

Correspondence: Jasvinder.md@gmail.com

${ }^{1}$ Medicine Service, VA Medical Center, 510, 20th street South, FOT 805B, Birmingham, AL 35233, USA

${ }^{2}$ Department of Medicine at School of Medicine, University of Alabama at Birmingham, 1720 Second Ave. South, Birmingham, AL 35294-0022, USA

Full list of author information is available at the end of the article

C C The Author(s). 2021 Open Access This article is licensed under a Creative Commons Attribution 4.0 International License, which permits use, sharing, adaptation, distribution and reproduction in any medium or format, as long as you give appropriate credit to the original author(s) and the source, provide a link to the Creative Commons licence, and indicate if changes were made. The images or other third party material in this article are included in the article's Creative Commons licence, unless indicated otherwise in a credit line to the material. If material is not included in the article's Creative Commons licence and your intended use is not permitted by statutory regulation or exceeds the permitted use, you will need to obtain permission directly from the copyright holder. To view a copy of this licence, visit http://creativecommons.org/licenses/by/4.0/. The Creative Commons Public Domain Dedication waiver (http://creativecommons.org/publicdomain/zero/1.0/) applies to the data made available in this article, unless otherwise stated in a credit line to the data. 
Discussion: The study will assess the efficacy of a behavioral intervention to improve ULT adherence in minority populations with gout.

Trial registration: ClinicalTrials.gov NCT 02741700. Registered on 14 September 2018

Keywords: Gout, Storytelling intervention, Behavioral intervention, Randomized trial, African American, Racial/ ethnic minorities

\section{Introduction}

Medication non-adherence, i.e., not taking medications as prescribed, costs over $\$ 100$ billion a year in excess hospitalizations in the USA [1]. The problem is worse in patients with gout, with only $37 \%$ of gout patients taking $80 \%$ of their prescribed medication in the first 12 months of the treatment [2]. Gout is the most common inflammatory arthritis. It affects 8.3 million Americans [3] and $5 \%$ of veterans [4] Its prevalence is increasing [3]. Multiple patient- and system-related barriers contribute to medication non-adherence $[5,6]$. In a Cochrane systematic review, only 18 of 58 medication adherence interventions led to improved outcomes in chronic disease [7]. The successful interventions incorporated self-efficacy or peer group, i.e., were more patient-centered. Thus, more efficacious, feasible, lowcost behavioral interventions are needed to address nonadherence in chronic disease management, especially in gout.

Compared to Whites, Blacks or African Americans (AAs) have higher gout prevalence and incidence $[3,8]$ and worse outcomes, including higher serum urate (SU) and higher rate of emergency room visits or hospitalizations for gout [9]. Specifically, AAs are more nonadherent with urate-lowering therapy (ULT) [10] and lower rates of treatment with allopurinol, the most commonly used ULT [11]. A higher ULT non-adherence in AAs is related to patient knowledge gaps, perceptions, and perceived barriers to gout treatment [12]. Poor gout outcomes are also partially attributable to higher rates of hypertension [8], obesity, diabetes, and renal failure in AAs compared to Whites [13]. Thus, gout leads to a disproportionately higher disease burden in AAs compared to Whites.

A recent 6-month study using a patient storytelling intervention about their experience with the disease and its treatment showed improved hypertension control in AAs with hypertension [14]. The hypertension study represented a model for chronic asymptomatic diseases. Gout represents a model for chronic, intermittently symptomatic diseases, due to its well-elucidated biochemical abnormality, pathophysiology, treatment, and outcomes, all related through a single factor, SU. Because gout is associated with symptoms, we anticipate that storytelling may impact adherence in a manner that is different from patients with hypertension [14]. Gout presents a relatively simple model in which the gold standard surrogate for disease outcomes, $\mathrm{SU}$, is primarily affected by ULT medication adherence $[15,16]$. Therefore, we designed a 12-month, multi-center, randomized controlled trial (RCT) to assess the effect of a culturally appropriate gout storytelling in AA veterans with gout. This manuscript provides the protocol for this randomized trial.

The main objective of the randomized study is to assess the efficacy of a culturally appropriate gout storytelling in AA veterans with gout. We hypothesize that gout storytelling would improve ULT adherence more than the control intervention in AA veterans with gout.

\section{Methods \\ Study objective}

The primary objective of this 1-year study is to assess the efficacy of a novel gout storytelling in patient's own voice on ULT adherence in gout, assessed by an objective measure of ULT adherence assessed with MEMSCap $^{\mathrm{ma}}$ electronic bottlecap monitoring at 6 months (primary trial end point) (Fig. 1). Secondary objectives are to assess patient outcomes including gout flare rate, patient satisfaction, target $\mathrm{SU}<6 \mathrm{mg} / \mathrm{dl}$ achievement, health-related quality of life (HRQOL), self-reported ULT adherence, and patient understandability of the intervention.

\section{Study overview}

Patients in the storytelling intervention group will view the designed storytelling video, and the usual care group will view a presentation on stress management for the same time duration (attention control), on an IPAD/ touchpad in the clinic during the baseline visit. Mailed DVDs to the enrolled patients (or in-person at previous visits, per patient preference) at 2 and 4 months with additional storytelling clips will reinforce messages for behavioral change received during the baseline visit through storytelling videos; mailed DVDs to the attention control group will provide information on stress management. We will use the Medication Event Monitoring System Cap (MEMSCap ${ }^{\text {tw }}$; Aprex Corp., Fremont, CA), a medication bottle cap with a microprocessor that records the occurrence and date and time whenever a 


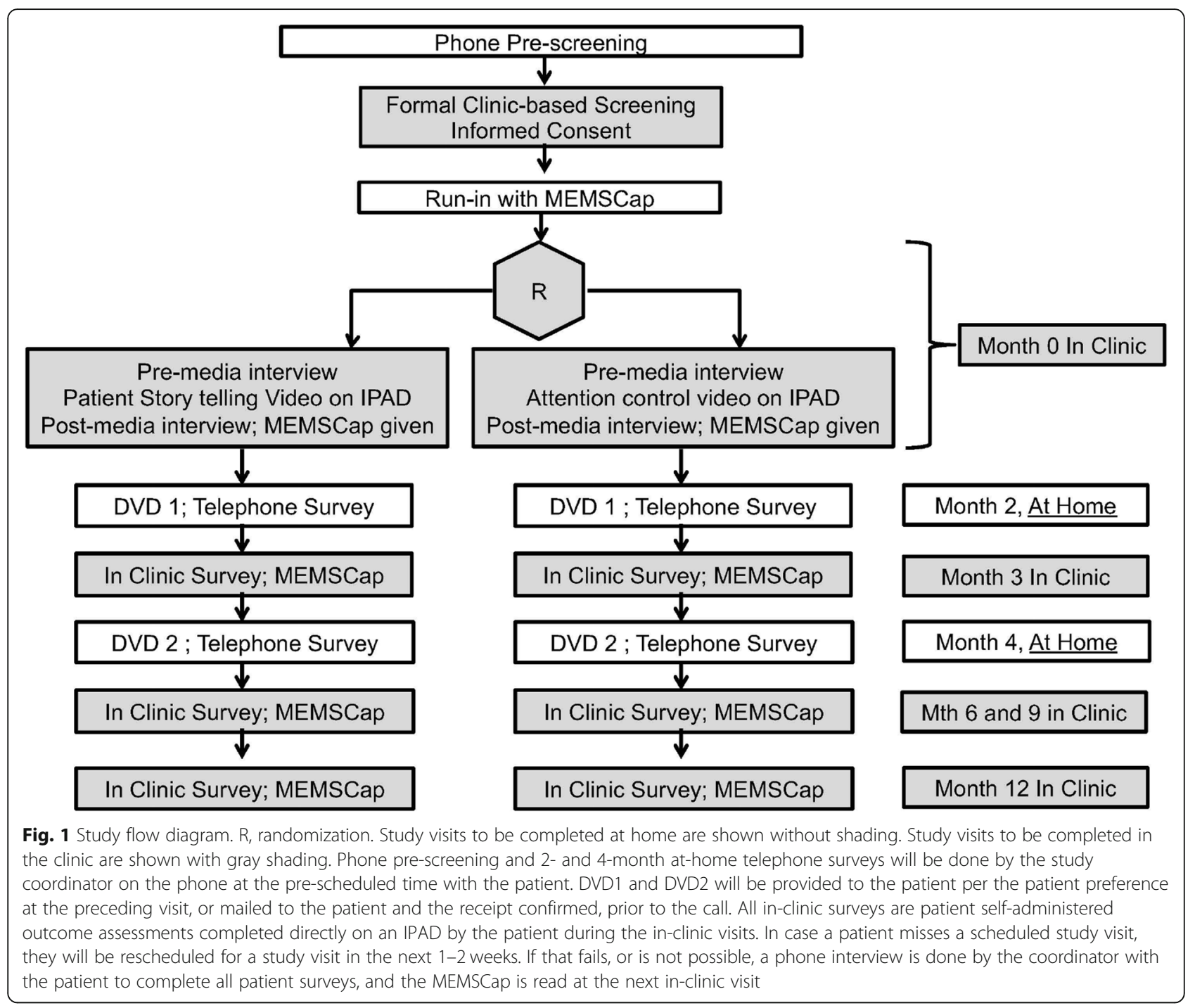

patient opens a vial using integrated microcircuits, as a measure of the primary outcome of ULT adherence [17]. It will be dispensed at the baseline visit, and data downloaded at each in-person visit. Patients will receive the first 90-day supply in special bottles with MEMSCap ${ }^{\text {тм }}$ and be trained at the initial visit regarding its importance and to bring it to the 3-, 6-, 9-, and 12-month clinic visits; opening/closing of the bottle cap will be demonstrated to participants.

\section{Study population, study sites, randomization, and ClinicalTrials.gov registration}

We will conduct a multicenter, parallel 2-arm, 12-month open-label RCT comparing a culturally appropriate gout-storytelling intervention to a control intervention (stress reduction) in AA veterans with gout. We will recruit participants at Birmingham, Philadelphia, and St. Louis Veterans Affairs (VA) Medical Centers (VAMCs).
Specific aims

Our specific aims (SAs) are to assess the efficacy of a gout storytelling intervention for the following:

SA1: Improving ULT adherence, directly measured by using MEMSCap ${ }^{\mathrm{TM}}$ at 3, 6, 9 months (assess the intervention's effect), and 12 months (assess the durability of effect)

SA2: Improving gout flare rate, patient satisfaction, target $\mathrm{SU}<6 \mathrm{mg} / \mathrm{dl}$ achievement, health-related quality of life (HRQOL), self-reported ULT adherence, and patient understandability of the intervention, as important gout outcomes.

\section{Intervention platform}

The intervention will be delivered in a series of three videos in the proposed RCT (details below) at baseline using an IPAD and at 2- and 4-month DVDs sent via mail or provided in-person to the participant at the 
baseline and follow-up visits, per patient preference. Each video will either have the gout-storytelling intervention with patient narrated stories and "learn more" gout material in a patient's own voice or the stress management video. The duration of the videos is the same (15-20 min). DVDs will have new stories from our storytelling stars, based on patient preference for messages (diet, medication, disease impact), but will be like those presented at the baseline visit.

Parallel study protocols for the intervention and comparison groups have been designed. Specifically, the contact with research personnel (including rapport building), duration of the intervention, mailings and phone calls, and the assessment schedules will be identical in the intervention versus attention control arms (Fig. 1).

\section{Study sites, personnel training, Institutional Review Board} (IRB) approval, and trial registration

Birmingham, Philadelphia, and St. Louis VAMCs are the three study sites. After the approval for our study from each IRB, experienced research associates will be responsible for patient recruitment, informed consent, and randomization procedures. Trained study research assistants at each site will be supervised by the respective site PI. The study is registered on ClinicalTrials.gov website (NCT 02741700).

\section{Study advertisement for participation}

We will advertise our study in three ways, like our previous studies: (1) via IRB-approved mailed letters to patients with gout who preliminarily meet the study eligibility criteria based on the VA pharmacy, administrative, and clinical database records and (2) through emails to the primary care physicians at regular intervals, every $2-3$ months.

\section{Identification of the study population and pre-screening using pharmacy records and a phone call}

Our study population is AA veterans with gout with low adherence, defined as an average ULT MPR $<0.80$ (= \#days of outpatient days' ULT supply used/\#days' supply), based on the immediately prior period of 180 days (low adherers). Veterans will be identified with the help of the Automated Data Package Application Coordinator (ADPAC) at each facility. Using the VISTA pharmacy query, the research assistant at each VA site will obtain an automated monthly query in VISTA pharmacy records to capture lists of veterans at each VA site every week who filled a recent ULT (allopurinol or febuxostat) prescription, along with the dates of ULT refills in the last 6-9 months and days' supply, to identify potentially eligible patients. Baseline adherence assessment using databases is different than primary outcome assessment with MEMSCAP ${ }^{\text {mi }}$ due to feasibility. Knowing that the race/ethnicity variable is not recorded on all and sometimes recorded incorrectly in VA databases, we will preliminarily ascertain it from the patient's inpatient and outpatient CPRS medical record and confirm it during phone pre-screening. The research assistant at each site will call and pre-screen eligible veterans for inclusion/ exclusion criteria using the pre-screening questionnaire. Those that pass the pre-screen will be invited to come for a study screening and enrollment visit.

\section{Subject eligibility criteria for the STRIDE-GO study}

The inclusion criteria are as follows: (1) AA veterans (self-identified race will be the gold standard; bi- and multi-racial included) aged 18 years or above with a diagnosis of gout (1 inpatient or $\geq 2$ outpatient International Classification of Diseases, ninth revision, clinical modification [ICD-9-CM] codes 274.x or 274.xx) [4]; (2) meet the 1977 Preliminary American College of Rheumatology (ACR) classification criteria for gout [18]; (3) currently prescribed and filled oral ULT medication prescription (allopurinol or febuxostat) for at least 6 months; (4) ULT MPR $<0.80$ for the preceding 6 months excluding the most recent filled ULT prescription for which the period of prescription fill $(90,30$, or 60 days) has not been completed (the most common VA prescription is 90 days-see the "Protocol modifications" section); and (5) able to provide informed consent.

The exclusion criteria are as follows: (1) ULT MPR $\geq$ 0.80 (see the "Protocol modifications" section) and (2) patients who must redistribute daily pill into the pillbox.

The drop-out criteria are as follows: (1) patient refusal to continue to participate in the trial and (2) patient starting the use of a pillbox for the ULT.

\section{Subject screening, in-clinic enrollment, and baseline study assessments}

For the in-clinic screening and baseline visit, patients will meet the research associate at their regular clinic location and, once their scheduled clinical visit ends, walk to a private area for more detailed screening and enrollment. Once enrolled in the study, study participants will meet the research associate at the study clinic location, if they are coming for the study visit only. Study participants will provide informed consent and HIPPA authorization. The site research associate will screen the veterans for study eligibility criteria, including the preliminary ACR gout classification criteria. For those who meet all the study inclusion criteria, the site PI will confirm eligibility for enrollment in the study.

Patients will then complete the baseline patient assessments including the following: (1) demographics-age, gender, income (covariates), and marital status; (2) gout duration, baseline frequency of gout flares requiring 
treatment, and baseline patient satisfaction with ULT treatment; (3) baseline gout-specific HRQOL assessment using the gout assessment questionnaire (GAQ); (4) alcohol use and body mass index (BMI) (covariates; alcohol and higher BMI are associated with a higher risk of incident gout, gout flares, and higher SU level [3, 19-21]); (5) blood draw for baseline SU; and (6) ULT non-adherence on the self-reported questionnaire by Voils et al. [22]. Additional information will be obtained including contact information, best time to contact, and email address. Veterans will be provided with a \$25 remuneration for completing the study assessments at the baseline visit. Patients without DVD players will be provided with a DVD player to watch the DVDs at home at 2 and 4 months.

\section{Randomization and allocation to treatment and allocation concealment}

Once patients complete all baseline assessments, they will be randomized into one of the two groups, gout storytelling versus control intervention. Randomization will be based upon a permuted variable block design. An online computerized simple randomization scheme will be programmed by a study biostatistician for each VA site with redundant systems established to avoid interruption during periods such as server upgrades and maintenance, available through a secure Internet link. This ensures that the allocation is concealed from the study assessors and the stud PI.

Due to the nature of interventions, patients will be aware of the group assignments. The two treatment groups are as follows:

\section{Group 1: Storytelling intervention \\ Group 2: Usual care (attention control)}

The intervention group will view the storytelling video modules in entirety at the baseline visit (in-person) and provided DVDs to view study month 2 (by mail/previous visit) and study month 4 (by mail/previous visit). Storytelling in African American veterans' own voices will focus on improving ULT adherence, along with patient-narrated video segments about gout and its treatment under "Learn More," by adapting a pre-tested power-point slide presentation narrated by a veteran with gout. The intervention group will also get a printed copy of the stories and the power-point presentation in the "Learn More" section at baseline. Each intervention installment will present new stories and Learn More gout content. Participants will be introduced to the MEMSCap ${ }^{\mathrm{Tm}}$ and trained during their initial visit by research assistants.

The usual care comparison group will receive the attention identical to the intervention condition (attention control), aside from not including the gout storytelling modules. The usual care group will view a stress management video, based on the content adapted from the Centers for Disease Control in a power-point presentation and narrated by the same veteran, who narrated the gout power-point presentation. The video segments will be of the same length as in the intervention group.

\section{Patient recruitment and retention}

We will stay connected with the study participants during the follow-up with mailed postcards and phone call reminders. We will mail study newsletters featuring images and quotes from consenting participants, after appropriate permissions from each site's IRB. Study retention rates in the previous studies conducted by the PI that included minorities have exceeded $80 \%$ [23, 24]. Our current protocol allows for a dropout of similar magnitude, as the worst-case scenario. However, based on our experience and expertise, we expect the dropout rate to be lower.

\section{Study procedures at follow-up visits and data collection tools, including the 12-month blood draw}

Follow-up assessments will be done at 3, 6, and 9 months after the baseline visit lasting $30 \mathrm{~min}$ in-person, 1 month via phone lasting $15 \mathrm{~min}$ and at 12 months lasting $1 \mathrm{~h}$ in person. One-month follow-up will be done via a telephone-administered survey at the patient's convenience in their home. The 3-, 6-, and 9-month visits will be in-person, and MEMSCap ${ }^{\text {Tx }}$ data will be downloaded and other outcomes (gout flares, GAQ, and selfreported medication adherence) captured at each visit (Table 1). The schedule of collection of various outcomes is shown in Table 2. Post-card (and emails, when applicable) will be mailed 1 week and phone call made 2 days prior to the follow-up to remind the patient of the follow-up assessments. To minimize patient responder burden, veterans are only completing assessments related to primary and select secondary study outcomes (including a 4-item gout flare questionnaire) at each study follow-up visit.

The 12-month visit (end-of-study) will be in-person, similar to the baseline visit, and include patient satisfaction with treatment and SU assessment. MEMSCap ${ }^{\mathrm{m}}$ data will be downloaded, and veterans will complete a questionnaire including gout flares, GAQ, and selfreported medication adherence, as in 3-, 6- and 9-month follow-up. Patient satisfaction with treatment and SU will be done only at the 12-month follow-up to reduce patient burden (expected to take an additional 30-min). Veterans unable to come to the study clinic for the 12month visit will be offered SU blood draw at their nearest community-based VA outpatient clinic (CBOC; a routine laboratory test), and the research associate will administer 12-month assessments via the phone interview. 
Table 1 Summary of primary and secondary outcomes and outcome measures to achieve SA1 and SA2

\begin{tabular}{|c|c|c|}
\hline & Description & Clinically meaningful change \\
\hline \multicolumn{3}{|c|}{ Primary outcome/follow-up time of assessment (SA1) } \\
\hline $\begin{array}{l}\text { 6-month ULT medication adherence measured } \\
\text { using MEMSCap }{ }^{\mathrm{T} *} / 3,6,9 \text {, and } 12 \text { months }\end{array}$ & $\begin{array}{l}\text { Medication adherence to ULT measured using } \\
\text { MEMSCap }{ }^{\text {TM }}[25]\end{array}$ & $\begin{array}{l}\text { Absolute difference of } 6 \% \text { between the } \\
\text { groups representing a medium effect size } \\
\text { of } 0.40 \text { (a smaller difference is unlikely to } \\
\text { be meaningful) }\end{array}$ \\
\hline \multicolumn{3}{|l|}{ Secondary outcomes (SA2) } \\
\hline Number of gout flares $/ 3,6,9$, and 12 months & $\begin{array}{l}\text { Number of gout flares in the last } 1 \text { and } 2 \text { months } \\
\text { Current flare: } 4 \text {-item patient-reported assessment } \\
\text { of gout flare [26] }\end{array}$ & $\begin{array}{l}20 \% \text { fewer patients with gout flares needing } \\
\text { treatment (absolute difference) }\end{array}$ \\
\hline $\begin{array}{l}\text { Patient Satisfaction with Medications } \\
\text { Questionnaire (SATMED-Q)/6 and } 12 \text { months }\end{array}$ & 17-item patient-reported with six dimensions [27] & $\begin{array}{l}\text { Total score: } 5.9 \text { to } 13.4 \text { points SATMED-Q } \\
\text { domain scores: } 5.9 \text { to } 20.6 \text {, with most } \\
\text { estimates close to } 10 \text { [28] }\end{array}$ \\
\hline Serum urate $<6 \mathrm{mg} / \mathrm{dl} / 12$ months & Serum urate standard biochemical assay [29] & $\begin{array}{l}20 \% \text { more patients achieving target serum } \\
\text { urate }<6 \mathrm{mg} / \mathrm{dl} \text { (absolute difference) }\end{array}$ \\
\hline $\begin{array}{l}\text { Patient Education Materials Assessment Tool } \\
\text { for Audiovisual Materials (PEMAT-AV)/2 and } \\
4 \text { months }\end{array}$ & $\begin{array}{l}\text { Understandability (16 items), accountability } \\
\text { (4 items), and potential impact of various } \\
\text { messages on change in behavior, including } \\
\text { ULT adherence [30] }\end{array}$ & No defined threshold \\
\hline $\begin{array}{l}\text { Gout-Specific Health-Related Quality of Life } \\
\text { (HRQOL) on Gout Impact Scale (GIS) of the } \\
\text { Gout Assessment Questionnaire (GAQ)/3, 6, } \\
9 \text {, and } 12 \text { months }\end{array}$ & $\begin{array}{l}\text { A validated measure of specific impact of gout on } \\
\text { HRQOL [23]; } 22 \text { items ( } 0-100 \text { scale) that constitute } \\
5 \text { subscales }\end{array}$ & $\begin{array}{l}\text { A clinically important difference of the GIS } \\
\text { is between } 5 \text { and } 8 \text { points on each GIS } \\
\text { subscale [31] }\end{array}$ \\
\hline $\begin{array}{l}\text { Self-reported ULT adherence by Voils et al./3, } 6 \text {, } \\
9 \text {, and } 12 \text { months }\end{array}$ & $\begin{array}{l}\text { A validated questionnaire [22] with } 2 \text { scales, } \\
\text { measuring the extent of non-adherence ( } 3 \text { items) } \\
\text { and the reasons for non-adherence ( } 21 \text { items) }\end{array}$ & No defined threshold \\
\hline
\end{tabular}

${ }^{*}$ Analyses of 12-month MEMSCAP ${ }^{\mathrm{TM}}$ will indicate sustenance of the treatment effects noted at 6 months

The primary time point for analyses will be at 6 months. Assessments are kept short considering the responder burden and to ensure study retention. Veterans will be provided with a $\$ 25$ remuneration for completing each study assessment; an additional $\$ 25$ will be provided to those who perform both blood draws at baseline and 12-month end-of-study visits.

\section{Data management, quality assurance, and monitoring} Data management and quality assurance are particularly important for this study. All data collection will occur using HIPAA-compliant Microsoft Access ${ }^{\circledR}$ database or the VA Research Electronic Data Capture (REDCap; Nashville, TN) database on a secure VA server behind a firewall. Data entered by veterans at screening, baseline, and follow-up visits using the touchscreen computers will be directly captured in the study database. The study biostatistician will coordinate and oversee data management. Since personal identifiers will be collected, all database versions will be stored on the VA server. We will program logic and range checks in SAS 9.2 (SAS Cary, NC) to ensure timely identification of data fields requiring querying and clarification. A frequent multiple-backup strategy is proposed due to our desire not to lose any study data.

Table 2 Schedule of visits and timing of each data point collection

\begin{tabular}{|c|c|c|c|c|c|c|}
\hline & Baseline visit & $\begin{array}{l}\text { Telephone visits } \\
\text { ( } 2 \text { and } 4 \text { months) }\end{array}$ & 3-month visit & 6-month visit & 9-month visit & 12-month visit \\
\hline MEMSCap ${ }^{\text {TM* }}$ adherence & $x$ & & $\mathrm{X}$ & $x$ & $x$ & $\mathrm{X}$ \\
\hline \multicolumn{7}{|l|}{ Secondary } \\
\hline Number of gout flares & $x$ & & $\mathrm{X}$ & $x$ & $x$ & $x$ \\
\hline SATMED-Q & $x$ & & & $\mathrm{x}$ & & $\mathrm{X}$ \\
\hline Serum urate & $x$ & & & & & $\mathrm{x}$ \\
\hline PEMAT-AN & $x$ & $x$ & & & & \\
\hline GIS-GAQ & $x$ & & $x$ & $x$ & $\mathrm{X}$ & $\mathrm{x}$ \\
\hline Self-reported ULT Adherence & $\mathrm{X}$ & & $\mathrm{x}$ & $\mathrm{x}$ & $\mathrm{X}$ & $\mathrm{x}$ \\
\hline
\end{tabular}


Description of outcomes, outcome measures, and covariates (SA1 and SA2)

\section{Choice of outcomes}

All proposed primary and secondary study outcomes are clinically meaningful and patient-centered (Table 1). The primary outcome is ULT adherence at 6 months measured by MEMSCap ${ }^{\mathrm{TM}}$. Secondary outcomes are gout flares, patient satisfaction with treatment, SU, goutspecific health-related quality of life (HRQOL), and patient education materials assessment.

\section{Primary outcome (SA1)}

ULT adherence We will calculate 3-, 6-, 9-, and 12month ULT adherence using the MEMSCap ${ }^{\mathrm{Tm}}$. MEMS$\mathrm{Cap}^{\mathrm{TM}}$ is more accurate and has a higher validity compared to other measures of adherence (self-report, claims, etc.) [25], with excellent internal reliability (Cronbach's alpha $=0.94$ ), [32] high degree of agreement with pill counts (kappa $=0.72$ ) [32, 33], and high predictive validity, given the association with lower symptom severity [32]. Despite some limitations, it is considered the best objective measure of medicationtaking behavior. MEMSCap ${ }^{\mathrm{TM}}$ is child-resistant and wirelessly transfers the dosing data when used in conjunction with a MEMSCap ${ }^{\mathrm{Tm}}$ reader. It can record and store up to 3800 dosing events. The mean ULT adherence at the 6month period will be calculated. After careful consideration, we decided not to include probenecid, since it is given twice daily and constitutes only $5 \%$ of all ULTs prescribed. Allopurinol and febuxostat, ULTs taken once daily, will be included in this study.

\section{Secondary outcomes (SA2)}

Patient-reported gout flares This will be assessed by the self-reported total number of gout flares in the last 1 and 2 months.

A validated gout flare will be assessed for patientreported gout flare, based on the combination of a patient report of a gout flare along with the presence of any patient-reported warm joint, any patient-reported swollen joint, and patient-reported pain at rest score of $>3$ (0-10 scale), a flare definition developed and validated [26]. This takes $<5 \mathrm{~min}$ to complete.

Patient satisfaction with treatment Patient satisfaction will be assessed by the Satisfaction with Medications Questionnaire (SATMED-Q) [27]. It is designed for use with patients presenting with any chronic illness and taking any type of prolonged pharmacological treatment. SATMED has 17 items with six dimensions: treatment effectiveness, convenience of use, impact on daily activities, medical care, global satisfaction, and side effects adapted for our study. Responses are scored on a Likert scale from 0 to 4 , as follows: $0=$ "no, not at all"; 1 = "a little bit"; 2 = "neither a lot, nor a little"; 3 = "quite a lot"; and 4 = "yes, very much." The total score ranges from 0 to 68 , transformed to a $0-100$ scale for ease of understanding. It takes $<5 \mathrm{~min}$ to complete. Thresholds for minimal clinical significant effect are shown in the table, i.e., for the total score, they were 5.9 to 13.4 points, and for the SATMED-Q domain, scores were 5.9 to 20.6, with most estimates close to 10 [29].

Serum urate Serum urate will be determined by an enzymatic uricase method manufactured by Stanbio Laboratory (Boerne, TX), a standardized assay [29]. This biochemical outcome is a surrogate for disease control and the key target of ULT used by regulatory authorities for gout drug approval. Achieving and maintaining serum urate $<6 \mathrm{mg} / \mathrm{dl}$ ("target") is associated with a lower risk of gout flares, tophi, and medical care costs [34-36], outcomes that are relevant to patients and providers, as well as the health care system.

Self-reported ULT adherence by Voils et al. Self-reported medication adherence will be assessed using a validated questionnaire [22]. It has two scales, one measuring the extent of non-adherence (3 items scored from strongly disagree $($ score $=1$ ) to strongly agree (score = 5) to produce an aggregate score (higher scores indicate greater levels of non-adherence)) and the other measuring the reasons for non-adherence (21 items scored from not at all $($ score $=1)$ to very much $($ score $=5$; no total score calculated)). Intraclass correlations were 0.58 for the extent score and ranged from 0.07 to 0.64 for the reasons. It takes $<5 \mathrm{~min}$ to complete.

Gout-specific health-related quality of life (HRQOL) Gout-specific health-related quality of life (HRQOL) will be assessed with the Gout Impact Scale (GIS) of the Gout Assessment Questionnaire (GAQ), a validated measure of the specific impact of gout on HRQOL [23]. GIS contains 22 questions (0-100 scale) that constitute 5 subscales. The clinically important difference of the GIS is between 5 and 8 points [31]. It takes $5 \mathrm{~min}$ to complete and will be done at 0 and 12 months.

Patient Education Materials Assessment Tool for Audiovisual Materials (PEMAT-A/V) Patient Education Materials Assessment Tool for Audiovisual Materials (PEMAT-A/V) will be used to assess the understandability, accountability, and potential impact of various messages on change in behavior, including ULT adherence [30]; 16 items to assess understandability and 4 items for accountability are scored as agree $=1$ and disagree $=0$, with options for not applicable for some. We 
have adapted the scale to explore which material (stories vs. didactic) likely impacted behavior and which behavior changed because of the intervention. It will be done at 0 and 6 months.

\section{Covariates}

The following covariates will be assessed: (1) demographics-age, sex, marital status, and income; (2) gout duration and baseline gout flares; (3) body mass index (BMI; VA database); (4) alcohol use; and (5) baseline ULT MPR and baseline SU. We will analytically adjust for these covariates if they are not balanced by randomization.

\section{Statistical methods}

For both aims 1 and 2, descriptive statistics for demographics (age, income, marital status, gout disease duration) and clinical parameters (ULT adherence, \# gout flares, satisfaction, serum urate, GAQ) will be calculated. Specifically, central tendency measures (sample mean/ median for continuous measures, proportions for categorical measures), dispersion measures (variance, range), and precision ( $95 \%$ confidence intervals) will be calculated. Given the two-arm study design (storytelling vs. usual care), statistical procedures appropriate for two-group comparisons (two-sample $t$-tests, tests of proportions, ordinary least square regression, logistic regression, Poisson regression methodology) will be utilized to conduct crude as well as adjusted comparisons based upon the distributional nature of the outcome. For ULT adherence, an unadjusted analysis will be conducted using the two-sample $t$-test. To control for age, sex, income, alcohol use, and other covariates, ordinary least squares regression will be used to test for treatment differences after adjusting for covariates. If the normality assumption is violated, nonparametric methods will be used, instead of the parametric tests. Similar approaches will be used to test for differences in patient satisfaction and HRQOL.

Poisson or quasi-Poisson regression will be used to test for group differences in gout flare rates in the last 1 or 2 months, while adjusting for covariates. Careful attention will be paid to the distributional assumptions for Poisson regression, and methods to adjust for overdispersion will be employed. Finally, separate logistic regression models will be used to measure treatment differences in the odds of achieving target serum urate $<6 \mathrm{mg} / \mathrm{dl}$. To analyze the longitudinal data $(3,6,9$, and 12 months), we will use mixed linear models for ULT adherence (continuous outcome) and generalized estimating equations for target serum urate $<6 \mathrm{mg} / \mathrm{dl}$ (categorical). All analyses will be guided by intent-to-treat analysis principles.

No interim analyses were planned.

\section{Sample size and power}

Hypothesis 1: We hypothesize that the mean ULT adherence will be higher in the intervention versus the comparison groups

Assuming a standard deviation (SD) of $15 \%$, similar to the SD of $14 \%$ reported by Briesacher et al. [2], 120 patients/group (total of 306 to account for 18\% drop out rate) will provide $80 \%$ power to detect an absolute difference between means of $6 \%$ for MEMSCap ${ }^{\mathrm{Tm}}$ ULT MPR, assuming a control group adherence of 55\%, intervention group mean of $61 \%$ (range, $0-100 \%$ ) [2], and using a two-tailed type I error rate of 0.05 . A difference of $6 \%$ equates to a medium Cohen's effect size of 0.40. For a larger SD of $20 \%$, we still have the power to detect between-group differences of $8 \%$ in MPR, which equals medium Cohen's effect size of 0.53 .

\section{Project management plan \\ Organizational structure}

The STRIDE-GO Coordinating Center will be at the BHM VAMC under the direction of Dr. Singh. The Coordinating Center will provide scientific and administrative coordination of the study. This includes the development of the study protocol, scheduling of meetings and calls, answering questions about the protocol, site visits, progress reports, and administration of funds.

\section{Performance standards and participant accrual requirements}

The Coordinating Center will set performance standards and monitor site activities to assure recruitment and retention rates, and data quality and protocol adherence standards are met. Under-performing sites and issues will be identified, and solutions recommended. Periodic conference calls will help in resolving issues through the process of sharing experiences.

\section{Adherence to protocol}

Strict adherence to the protocol is mandatory throughout the course of the study; any anticipated deviation from it should be discussed prospectively with the Coordinating Center. The Coordinating Center will monitor the number of approved and unapproved protocol exceptions at each site and report these in interim statistical reports. Serious protocol violations include failure to obtain a valid informed consent and erroneously withdrawing participants from the study.

\section{Data quality and audits}

Continuous monitoring of study data quality will be jointly performed by the Coordinating Center biostatistician, as completed de-identified data are transmitted from participating sites. The monitoring will include the frequency of data problems such as missing data, 
unusual values, and inconsistent data. The Coordinating Center will review data as they are received for accuracy, completeness, consistency between related data items, and adherence to the protocol. Data query reports will be sent to the participating sites for processing. Data quality summaries will be generated monthly by the Coordinating Center and reviewed. The Coordinating Center will communicate commonly occurring problems to all sites and work directly with specific sites where higher error rates are detected. The Coordinating Center will perform data audits if the data quality summary indicates data issues. These audits will follow standards used in clinical trials, including the comparison of case report forms with primary data sources.

\section{Timeline}

Aims 1 and 2 will be achieved in $0-45$ months and dissemination and implementation in 43-48 months (Table 3). This table also shows a plan for patient enrollment, followup assessments, and the study completion visit.

\section{Protocol modifications}

In this study, we made two protocol modifications, both prior to study initiation. First, we found inconsistency between pharmacy-based vs. patient self-reported ULT MPR use during screening. We also found that there was a discrepancy for baseline ULT MPR $<80 \%$ determination using pharmacy record-based ULT MPR, based on the period selected, 3 vs. 6 vs. 12 months. We determined that pharmacy records were an imperfect measure and differed from MEMSCap ${ }^{\mathrm{ru}}$, our primary outcome measure. Therefore, we added a 1-month run-in period using MEMSCap ${ }^{\text {tw }}$ prior to randomization and made this the measure of baseline ULT MPR rather than the pharmacy records of ULT prescription fill. Second, we found that many patients had allopurinol ULT MPR of $80 \%$ or higher during the 1-month run-in period. This indicated a possible Hawthorne effect of using MEMSCap ${ }^{\mathrm{Tx}}$ and study participation on ULT adherence that would result in the potential exclusion of at-risk patients. Therefore, we changed the study inclusion criteria to allow the enrollment of eligible subjects regardless of their 1-month run-in period ULT MPR value, and pre-specified that in addition to the main analysis, we would also perform an analysis of all study outcomes, in particular primary outcome, by baseline ULT MPR of $<80 \%$ vs. higher. Other protocol modifications were related to the correction of typographic errors and for clarifications on the protocol for the site coordinators and site principal investigators.

\section{Discussion}

ULT adherence in gout is low $[16,37,38]$. Therefore, effective interventions are needed to address this critical gap in gout care. We chose gout as a model of chronic disease with gout flares to test the efficacy of storytelling intervention for several reasons: (1) gout has a clear pathophysiology, and clinical features are linked to SU, which is the key abnormality; (2) the gold standard surrogate for disease outcomes in gout, $\mathrm{SU}$, is primarily affected by ULT medication adherence [15, 16]; and (3) in contrast to gout, COPD and CHF have complex physiochemical mechanisms of dysfunction, have multiple causes for exacerbations (e.g., seasonal variation, infection), and have multiple approaches to treatment (drug class, routes).

A key reason to conduct this study in AAs with gout was the relative lack of observational data in minorities with gout and an absolute lack of data on data from trials on this subgroup of patients. The burden of gout is higher in AAs compared to Whites $[3,8,9]$. We

Table 3 Study timeline

\begin{tabular}{|c|c|c|c|c|}
\hline Year & Year 1 & Year 2 & Year 3 & Year 4 \\
\hline IRB approvals, clinicaltrials.gov regis & & & & \\
\hline Aims 1 and 2. RCT of storytelling Inte & & & & \\
\hline Adapt protocols from previous studi & & & & \\
\hline Participant recruitment & & & & \\
\hline Intervention delivery $(0-, 2-$ and $4-\mathrm{m}$ & & & & \\
\hline Data collection $(0-, 2-, 3-, 4-, 6-$, 9- a & & & & \\
\hline Completion of 12-month follow-up & & & & \\
\hline Quality Assurance/Quality Improven & & & & \\
\hline Manuscript: RCT results & & & & \\
\hline Data Analysis & & & & \\
\hline Manuscript 3: RCT results & & & & \\
\hline Dissemination and Implementation & & & & \\
\hline
\end{tabular}


recognized that enrollment of AA racial minority with gout in a clinical trial would be challenging, given the history of research in minorities in the USA, the widely prevalent distrust of research in the AA community, and the physical proximity of Birmingham, Alabama to Tuskegee, which is where the infamous Tuskegee study was conducted [39, 40]. However, the lack of effective interventions for AAs with gout in contrast to a disproportionate burden of gout in AAs was the key motivations. Therefore, we first developed a culturally appropriate gout-storytelling intervention for AAs with gout and designed this RCT for AAs with gout.

A successful trial completion will establish several important milestones for disparity research in rheumatic diseases: (1) culturally appropriate disease-specific patient-centered feasible behavioral interventions can be successfully developed for the AA minority population, based on qualitative work in the target population [41]; (2) high-quality RCTs can be conducted in AAs with rheumatic diseases; and (3) if the trial result is positive, an effective behavioral intervention for improving ULT MPR will be available for AAs with gout. Even if the storytelling intervention does not improve ULT adherence, the technique may be usefully applied to model other patient behaviors, such as patient-provider communication, appointment keeping, and lifestyle changes.

Limitations of the study include the lack of inclusion of economic evaluation in this trial and the lack of an independent steering committee. Strengths include a randomized controlled trial design, inclusion of attention control, a trial that will enroll African Americans with gout, test a culturally relevant and culturally appropriate intervention that will be easy to use in the future, if found to be effective.

\section{Abbreviations}

AA: African Americans; STRIDE-GO: SToRytelling to Improve DiseasE outcomes in Gout; ULT: Urate-lowering therapy; SU: Serum urate or serum uric acid; HBM: Health belief model; MEMS: Medication Event Monitoring System; MPR: Medication possession ratio; SATMED-Q: Satisfaction with Medications-Questionnaire

\section{Supplementary Information}

The online version contains supplementary material available at https://doi. org/10.1186/s13063-021-05847-9.

Additional file 1. Appendix 1

\section{Acknowledgements}

The views in this article are solely the responsibility of the author and do not necessarily represent the views of the Department of Veterans Affairs. I thank Dr. Seth Eisen of the Washington University School of Medicine and St. Louis Veterans Affairs Medical Center, Dr. Kenneth Saag of the University of Alabama at Birmingham, and Dr. Jeroan Allison of the University of Massachusetts for their contributions to the study design.

\section{Author's contributions}

Study concept and design: Singh, Eisen, Saag. Data acquisition, analysis, and interpretation of the results: N/A. First draft of the manuscript: Singh. Critical revision of the manuscript for important intellectual content: Singh. Statistical analysis: N/A. Obtained funding: Singh. Administrative, technical, or material support: Singh. Study supervision: Singh. The author(s) read and approved the final manuscript.

\section{Funding}

The research reported in this article was funded through a Veterans Affairs Health Services Research and Development Award (IIR-13-314). Dr. Singh is supported by the resources and use of facilities at the Birmingham VA Medical Center, Birmingham, AL, USA.

The funding bodies did not play any role in the design; in the collection, analysis, and interpretation of the data; in the writing of the manuscript; and in the decision to submit the manuscript for publication.

\section{Availability of data and materials}

The study PI and biostatistician will have access to the trial dataset. We are ready to share the data with colleagues, after obtaining appropriate permissions from the Veterans Affairs Ethics Committee, in accordance with the HIPAA and Privacy policies.

\section{Declarations}

Ethics approval and consent to participate

The study will be approved by the Institutional Review Board (IRBs) at the Birmingham, St. Louis, and Philadelphia Veterans Affairs Medical Centers. All investigations were conducted in conformity with the ethical principles of research.

\section{Consent for publication}

Not applicable

\section{Competing interests}

There are no financial conflicts related directly to this study. JAS has received consultant fees from Crealta/Horizon, Medisys, Fidia, PK Med, Two labs Inc., Adept Field Solutions, Clinical Care options, Clearview healthcare partners, Putnam associates, Focus forward, Navigant consulting, Spherix, MedIQ, Jupiter Life Science, UBM LLC, Trio Health, Medscape, WebMD, Practice Point communications, and the National Institutes of Health and the American College of Rheumatology. JAS owns stock options in TPT Global Tech, Vaxart pharmaceuticals, Atyu biopharma, Adaptimmune Therapeutics, GeoVax Labs, Pieris Pharmaceuticals, Enzolytics Inc., Seres Therapeutics Tonix Pharmaceuticals Holding Corp., and Charlotte's Web Holdings, Inc. JAS previously owned stock options in Amarin, Viking, and Moderna Pharmaceuticals. JAS is on the Speaker's Bureau of Simply Speaking. JAS has received institutional research support from Zimmer Biomet Holdings. JAS received food and beverage payments from Intuitive Surgical Inc./Philips Electronics North America. JAS is a member of the executive of Outcomes Measures in Rheumatology (OMERACT), an organization that develops outcome measures in rheumatology and receives arms-length funding from 8 companies. JAS serves on the FDA Arthritis Advisory Committee. JAS is a member of the Veterans Affairs Rheumatology Field Advisory Committee. JAS is the editor and the Director of the UAB Cochrane Musculoskeletal Group Satellite Center on Network Meta-analysis. JAS previously served as a member of the following committees: member, the American College of Rheumatology's (ACR) Annual Meeting Planning Committee (AMPC) and Quality of Care Committees; the chair of the ACR Meet-the-Professor, Workshop and Study Group Subcommittee; and the co-chair of the ACR Criteria and Response Criteria subcommittee.

\section{Author details}

${ }^{1}$ Medicine Service, VA Medical Center, 510, 20th street South, FOT 805B, Birmingham, AL 35233, USA. ${ }^{2}$ Department of Medicine at School of Medicine, University of Alabama at Birmingham, 1720 Second Ave. South, Birmingham, AL 35294-0022, USA. ${ }^{3}$ Division of Epidemiology at School of Public Health, University of Alabama at Birmingham, 1720 Second Ave. South, Birmingham, AL 35294-0022, USA. 
Received: 26 June 2021 Accepted: 20 November 2021 Published online: 04 December 2021

\section{References}

1. Sokol MC, McGuigan KA, Verbrugge RR, Epstein RS. Impact of medication adherence on hospitalization risk and healthcare cost. Med Care. 2005;43(6): 521-30. https://doi.org/10.1097/01.mlr.0000163641.86870.af.

2. Briesacher BA, Andrade SE, Fouayzi H, Chan KA. Comparison of drug adherence rates among patients with seven different medical conditions. Pharmacotherapy. 2008;28(4):437-43. https://doi.org/10.1592/phco.28.4.437.

3. Zhu Y, Pandya BJ, Choi HK. Prevalence of gout and hyperuricemia in the US general population: the National Health and Nutrition Examination Survey 2007-2008. Arthritis Rheum. 2011;63(10):3136-41. https://doi.org/10.1002/a rt.30520.

4. Singh JA, Hodges JS, Toscano JP, Asch SM. Quality of care for gout in the US needs improvement. Arthritis Rheum. 2007;57(5):822-9. https://doi.org/1 0.1002 /art.22767.

5. Cramer J. Identifying and improving compliance patterns. In: Cramer JA, Spilker B, editors. Patient compliance in medical practice and clinical trials. New York: Raven Press; 1991. p. 387-92.

6. Zeber JE, Miller AL, Copeland LA, McCarthy JF, Zivin K, Valenstein M, et al. Medication adherence, ethnicity, and the influence of multiple psychosocial and financial barriers. Adm Policy Ment Health. 2011;38(2):86-95. https://doi. org/10.1007/s10488-010-0304-1.

7. Haynes RB, Yao X, Degani A, Kripalani S, Garg A, McDonald HP. Interventions to enhance medication adherence. Cochrane Database Syst Rev. 2005;4: CD000011. https://doi.org/10.1002/14651858.CD000011.pub2.

8. Hochberg MC, Thomas J, Thomas DJ, Mead L, Levine DM, Klag MJ. Racial differences in the incidence of gout. The role of hypertension. Arthritis Rheum. 1995;38(5):628-32. https://doi.org/10.1002/art.1780380508.

9. Coley K, Saul M, Pater K. Relationship between race, uric acid levels, uratelowering therapy and resource use in patients with gout. Arthritis \& Rheumatism. 2012;64(10 Suppl):S772.

10. Solomon DH, Avorn J, Levin R, Brookhart MA. Uric acid lowering therapy: prescribing patterns in a large cohort of older adults. Ann Rheum Dis. 2008; 67(5):609-13. https://doi.org/10.1136/ard.2007.076182.

11. Krishnan E, Lienesch D, Kwoh CK. Gout in ambulatory care settings in the United States. J Rheumatol. 2008;35(3):498-501.

12. Singh JA. Facilitators and barriers to adherence to urate-lowering therapy in African-Americans with gout: a qualitative study. Arthritis Res Ther. 2014 16(2):R82. https://doi.org/10.1186/ar4524.

13. Maynard W, Janet A, McAdams MN, Baer AC, Gelber A, Coresh J. Racial differences in gout risk and uric acid levels in both men and women in the Atherosclerosis Risk in Communities (ARIC) Study. [abstract]. Arthritis Rheum 2010;62 Suppl 10:1556

14. Mitcheli AJ, Selmes T. Why don't patients take their medication? Reasons and solutions in psychiatry. Adv Psyhiatric Treatment. 2007;13(5):336-46. https://doi.org/10.1192/apt.bp.106.003194.

15. Rashid N, Coburn BW, Wu YL, Cheetham TC, Curtis JR, Saag KG, et al. Modifiable factors associated with allopurinol adherence and outcomes among patients with gout in an integrated healthcare system. J Rheumatol. 2015;42(3):504-12. https://doi.org/10.3899/jrheum.140588.

16. Sarawate CA, Brewer KK, Yang W, Patel PA, Schumacher HR, Saag KG, et al. Gout medication treatment patterns and adherence to standards of care from a managed care perspective. Mayo Clin Proc. 2006;81(7):925-34. https://doi.org/10.4065/81.7.925.

17. Cramer JA, Mattson RH, Prevey ML, Scheyer RD, Ouellette VL. How often is medication taken as prescribed? A novel assessment technique. JAMA. 1989:261(22):3273-7. https://doi.org/10.1001/jama.1989.03420220087032.

18. Wallace SL, Robinson H, Masi AT, Decker JL, McCarty DJ, Yu TF. Preliminary criteria for the classification of the acute arthritis of primary gout. Arthritis Rheum. 1977;20(3):895-900. https://doi.org/10.1002/art.1780200320.

19. Choi HK, Atkinson K, Karlson EW, Curhan G. Obesity, weight change, hypertension, diuretic use, and risk of gout in men: the health professionals follow-up study. Arch Intern Med. 2005;165(7):742-8. https://doi.org/10.1 001/archinte.165.7.742.

20. Choi HK, Atkinson K, Karlson EW, Willett W, Curhan G. Alcohol intake and risk of incident gout in men: a prospective study. Lancet. 2004;363(9417): 1277-81. https://doi.org/10.1016/S0140-6736(04)16000-5.

21. Neogi T, Chen C, Niu J, Chaisson C, Hunter DJ, Zhang Y. Alcohol quantity and type on risk of recurrent gout attacks: an internet-based case-crossover study. Am J Med. 2014;127(4):311-8. https://doi.org/10.1016/j.amjmed.2 013.12.019.

22. Voils Cl, Maciejewski ML, Hoyle RH, Reeve BB, Gallagher P, Bryson CL, et al. Initial validation of a self-report measure of the extent of and reasons for medication nonadherence. Med Care. 2012;50(12):1013-9. https://doi.org/1 0.1097/MLR.0b013e318269e121.

23. Hirsch JD, Lee SJ, Terkeltaub R, et al. Evaluation of an instrument assessing influence of gout on health-related quality of life. J Rheumatol. 2008;35(12): 2406-14. https://doi.org/10.3899/jrheum.080506.

24. Singh JA, Mahowald ML, Noorbaloochi S. Intraarticular botulinum toxin A for refractory painful total knee arthroplasty: a randomized controlled trial. J Rheumatol. 2010;37(11):2377-86. https://doi.org/10.3899/jrheum.100336.

25. Greenlaw SM, Yentzer BA, O'Neill JL, Balkrishnan R, Feldman SR. Assessing adherence to dermatology treatments: a review of self-report and electronic measures. Skin Res Technol. 2010;16(2):253-8. https://doi.org/10.1111/j.16000846.2010.00431.x

26. Gaffo AL, Schumacher HR, Saag KG, Taylor WJ, Dinnella J, Outman R, et al. Developing a provisional definition of flare in patients with established gout. Arthritis Rheum. 2012;64(5):1508-17. https://doi.org/10.1002/art.33483.

27. Ruiz MA, Pardo A, Rejas J, Soto J, Villasante F, Aranguren JL. Development and validation of the "Treatment Satisfaction with Medicines Questionnaire" (SATMED-Q). Value Hēalth. 2008;11(5):913-26. https://doi.org/10.1111/j.1 524-4733.2008.00323.x

28. Rejas J, Ruiz MA, Pardo A, Soto J. Minimally important difference of the Treatment Satisfaction with Medicines Questionnaire (SATMED-Q). BMC Med Res Methodol. 2011;11(1):142. https://doi.org/10.1186/1471-2288-11-142.

29. Laboratory S. An EKF diagnostics company. Uric Acid LiquiColor ${ }^{\oplus}$ Test (Enzymatic) [online]. In. Boerne. TX Stanbio Laboratory.

30. Shoemaker SJ, Wolf MS, Brach C. Patient Education Materials Assessment Tool for Audiovisual Materials (PEMAT-AN). (Prepared by Abt Associates, under Contract No. HHSA2902009000121, TO 4). Publication No. 14-0002-EF. In. Agency for Healthcare Research and Quality: Rockville, MD; 2013.

31. Khanna D, Sarkin AJ. Minimally important differences of the gout impact scale in a randomized controlled trial. In: Khanna PP, et al. Rheumatology (Oxford): Minimally important differences of the gout impact scale in a randomized controlled trial; 2011.

32. Nakonezny PA, Byerly MJ, Rush AJ. Electronic monitoring of antipsychotic medication adherence in outpatients with schizophrenia or schizoaffective disorder: an empirical evaluation of its reliability and predictive validity. Psychiatry Res. 2008;157(1-3):259-63. https://doi.org/10.1016/j.psychres.2007. 05.001.

33. Brain C, Sameby B, Allerby K, Lindström E, Eberhard J, Burns T, et al. Twelve months of electronic monitoring (MEMS(R)) in the Swedish COAST-study: a comparison of methods for the measurement of adherence in schizophrenia. Eur Neuropsychopharmacol. 2014;24(2):215-22. https://doi. org/10.1016/j.euroneuro.2013.11.013.

34. Becker MA, Schumacher HR, MacDonald PA, Lloyd E, Lademacher C. Clinical efficacy and safety of successful longterm urate lowering with febuxostat or allopurinol in subjects with gout. J Rheumatol. 2009;36(6):1273-82. https:// doi.org/10.3899/jrheum.080814.

35. Halpern R, Fuldeore MJ, Mody RR, Patel PA, Mikuls TR. The effect of serum urate on gout flares and their associated costs: an administrative claims analysis. J Clin Rheumatol. 2009;15(1):3-7. https://doi.org/10.1097/RHU.0b013 e3181945d2c

36. Shoji A, Yamanaka $H$, Kamatani N. A retrospective study of the relationship between serum urate level and recurrent attacks of gouty arthritis: evidence for reduction of recurrent gouty arthritis with antihyperuricemic therapy. Arthritis Rheum. 2004;51(3):321-5. https://doi.org/10.1002/art.20405.

37. De Vera MA, Marcotte G, Rai S, Galo JS, Bhole V. Medication adherence in gout: a systematic review. Arthritis Care Res (Hoboken). 2014;66(10):1551-9. https://doi.org/10.1002/acr.22336.

38. Scheepers L, van Onna M, Stehouwer CDA, Singh JA, Arts ICW, Boonen A. Medication adherence among patients with gout: a systematic review and meta-analysis. Semin Arthritis Rheum. 2017;47(5):689-702. https://doi.org/1 0.1016/j.semarthrit.2017.09.007

39. Scharff DP, Mathews KJ, Jackson P, Hoffsuemmer J, Martin E, Edwards D. More than Tuskegee: understanding mistrust about research participation. J Health Care Poor Underserved. 2010;21(3):879-97. https://doi.org/10.1353/ hpu.0.0323.

40. Corbie-Smith G, Thomas SB, Williams MV, Moody-Ayers S. Attitudes and beliefs of African Americans toward participation in medical research. J 
Gen Intern Med. 1999;14(9):537-46. https://doi.org/10.1046/j.1525-1497.1 999.07048.x.

41. Singh JA, Herbey I, Bharat A, Dinnella JE, Pullman-Mooar S, Eisen S, et al.

Gout self-management in African American veterans: a qualitative

exploration of challenges and solutions from patients' perspectives. Arthritis

Care Res (Hoboken). 2017;69(11):1724-32. https://doi.org/10.1002/acr.23202.

\section{Publisher's Note}

Springer Nature remains neutral with regard to jurisdictional claims in published maps and institutional affiliations.

Ready to submit your research? Choose BMC and benefit from:

- fast, convenient online submission

- thorough peer review by experienced researchers in your field

- rapid publication on acceptance

- support for research data, including large and complex data types

- gold Open Access which fosters wider collaboration and increased citations

- maximum visibility for your research: over $100 \mathrm{M}$ website views per year

At $\mathrm{BMC}$, research is always in progress.

Learn more biomedcentral.com/submissions 